\title{
Gangrenous Stomatitis
}

National Cancer Institute

\section{Source}

National Cancer Institute. Gangrenous Stomatitis. NCI Thesaurus. Code C34852.

Gangrene of the mucous membranes of the mouth leading to ulcers and destruction of the soft tissues of the face and bones. It usually occurs in malnourished children in areas of poor sanitation and immunocompromised patients. 